\title{
Case Report \\ Cerebral Ischemia due to Aortic Arch Thrombosis Secondary to Iron Deficiency Anemia
}

\author{
Taha Nisar (D) and Rajanigandhi Hanumanthu \\ Department of Neurology, Rutgers New Jersey Medical School, Newark, New Jersey, USA \\ Correspondence should be addressed to Taha Nisar; tahanisar@hotmail.com
}

Received 15 April 2019; Accepted 2 July 2019; Published 18 July 2019

Academic Editor: Samuel T. Gontkovsky

Copyright (C) 2019 Taha Nisar and Rajanigandhi Hanumanthu. This is an open access article distributed under the Creative Commons Attribution License, which permits unrestricted use, distribution, and reproduction in any medium, provided the original work is properly cited.

\begin{abstract}
Thrombocytosis, hypercoagulable state, and hypoxia secondary to anemia are some of the mechanisms that are thought to cause strokes in patients with iron deficiency anemia (IDA). Several cases of middle-aged females with IDA who had embolic strokes due to aortic arch thrombosis have been reported. Majority of the cases were treated with anticoagulation. We report another case of embolic strokes in a patient with IDA treated with anticoagulation and iron replacement without recurrence of further episodes. We concluded that embolic phenomenon in middle-aged females with IDA warrants transesophageal echocardiogram with an evaluation of aortic arch.
\end{abstract}

\section{Introduction}

Iron deficiency anemia (IDA) is a cause of secondary thrombocytosis and commonly affects women of reproductive age [1]. The exact mechanism of thrombocytosis in the setting of IDA is unclear, and several theories exist [1,2]. Several cases of middle-aged females with IDA who had embolic strokes due to aortic arch thrombosis have been reported [2-4]. We present another interesting case of an embolic phenomenon in a middle-aged female with IDA, who had a thrombus visualized in the aortic arch on evaluation with a transesophageal echocardiogram (TEE), without evidence of atherosclerotic disease. Our patient was treated with anticoagulation and iron replacement without recurrence of further episodes.

\section{Case Report}

48-year-old African American woman with a history of menorrhagia presented to the emergency department with a complaint of a transient episode of weakness and numbness in her left arm and leg, lasting only 2 minutes. On presentation, her vitals were as follows: blood pressure: $151 / 81 \mathrm{~mm}$ $\mathrm{Hg}$, heart rate: 108 beats/minute, temperature: 98.2 degrees Fahrenheit, and respiratory rate: 20 . Upon presentation to the emergency department, her neurological examination was nonfocal. Her labs were suggestive of iron deficiency anemia (IDA) (Hb: $6.0 \mathrm{~g} / \mathrm{dl}, \mathrm{MCV}: 56.2 \mu \mathrm{m} 3$, platelets: 555 x1000 $\mu \mathrm{l}$, serum iron: $11 \mu \mathrm{l} / \mathrm{dl}$, total iron binding capacity (TIBC): $425 \mu \mathrm{g} / \mathrm{dl}$, ferritin: $4 \mathrm{ng} / \mathrm{ml}$, hemoglobin electrophoresis: $100 \%$ hemoglobin A, vitamin B 12, and folate were normal). She received a unit of packed red blood cells. Magnetic resonance imaging (MRI) of the brain showed acute infarctions in the right frontal lobe and left cerebellar hemisphere, as shown in Figure 1. There was a suspicion for embolic etiology given the bilateral location of the stroke, and the patient was started on anticoagulation. Transesophageal echocardiography (TEE) showed $0.9 \times 0.7[2-4] \mathrm{cm}$ echogenic density in the aortic arch, as shown in Figure 2. Computed tomography (CT) angiogram did not show any evidence of atherosclerotic disease but showed a $1.3 \mathrm{~cm}$ filling defect in the aortic arch, consistent with thrombus as shown in Figure 3. Repeat MRI of the brain 4 days later did not show any new strokes, and her neurological examination remained stable.

Further workup showed beta-2 glycoprotein IgM \& IgG, anticardiolipin IgM \& IgG, Sjogren Anti-SSA \& Anti-SSB, antiextractable nuclear AG (RNP, Smith), C3, C4 complements, protein $\mathrm{C}$, and factor II mutation within normal limits. MTHFR mutation was heterozygous in the A gene only, while homocysteine was normal. The patient was discharged on 


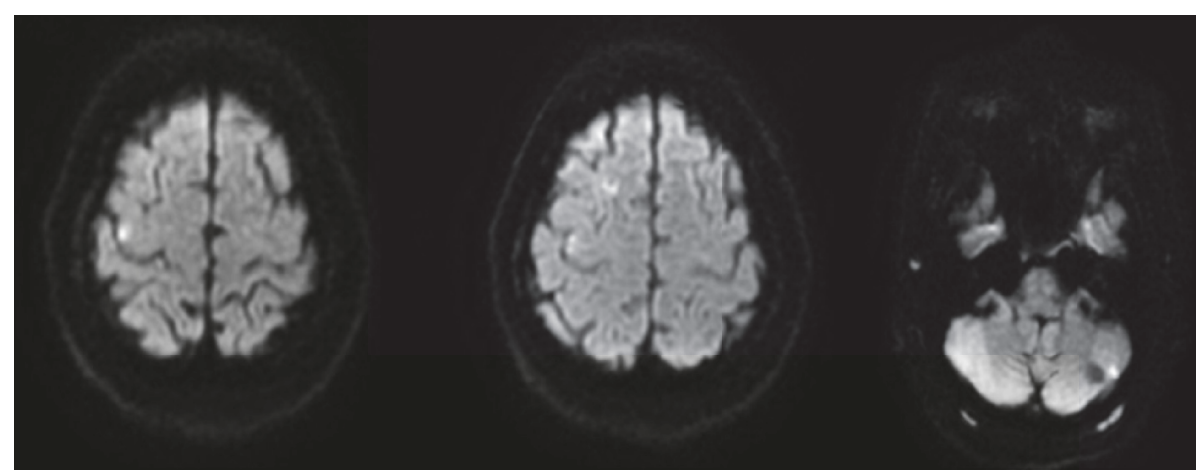

FIGURE 1: MRI of the brain without contrast showing small foci of diffusion restriction involving the right frontal lobe and left cerebellar hemisphere consistent with areas of acute infarctions. Abbreviation. MRI: magnetic resonance imaging.

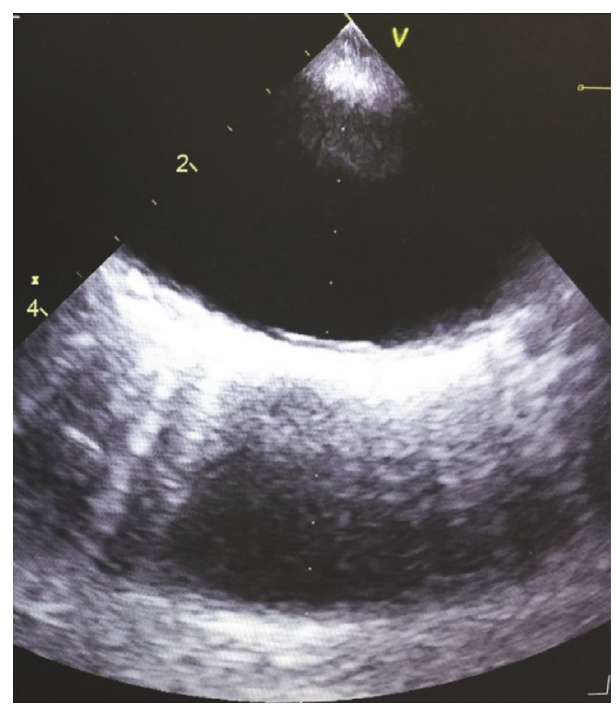

FIGURE 2: TEE demonstrating an echogenic density in the aortic arch, consistent with thrombus. Abbreviation. TEE: transesophageal echocardiogram.

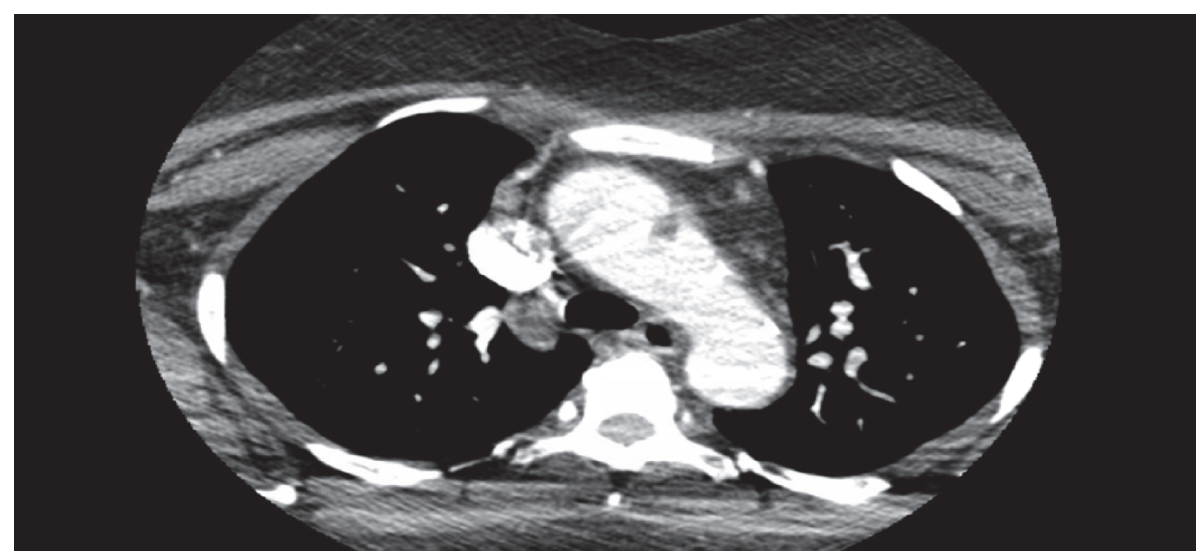

FIGURE 3: CT Angiogram of head and neck showing a $1.3 \mathrm{~cm}$ filling defect in the proximal aortic arch abutting the lateral wall of the aortic arch, consistent with thrombus. Abbreviation. CT: computed tomography. 


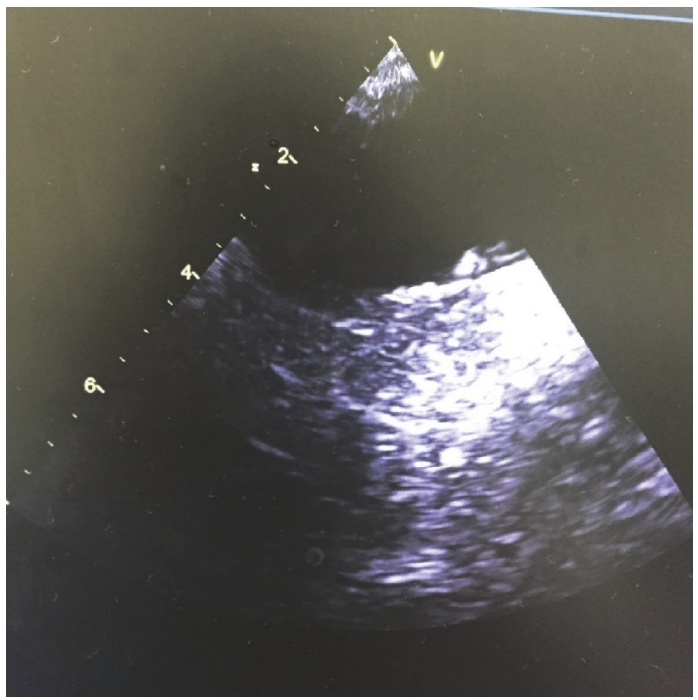

FIGURE 4: Follow-up TEE performed four months later demonstrating that prior echogenic mass in the aortic arch had decreased in size. Abbreviation. TEE: transesophageal echocardiogram.

warfarin, aspirin, and iron supplementation. Repeat TEE at four months showed that the prior echogenic mass in the aortic arch had decreased in size, as shown in Figure 4. She did not report further clinical events in eight-month followup and remained on warfarin and iron repletion.

\section{Discussion}

In 1990, Tunick et al. became the first to describe 3 cases of embolic strokes attributed to freely mobile aortic arch plaques that were visualized on a TEE. All the three patients reported had a severe atherosclerotic disease [5]. Aortic arch thrombosis is usually described in the context of severe atherosclerotic disease. However, in 1997, Laperche et al. published a case series of patients with recent arterial embolism due to mobile aortic arch thrombosis without diffuse aortic atherosclerotic debris [6]. Our patient had IDA and aortic arch thrombosis in the absence of atherosclerotic disease. However, the absence of macroscopic or iconographic evidence of atherosclerotic lesions does not eliminate the possibility of microscopic atherosclerotic lesions. Literature review shows several cases with a presentation similar to our patient, as shown in Table 1 [2-4]. The majority of the cases were treated with anticoagulation (heparin and warfarin), while one patient was treated with total arch replacement. Furthermore, several cases of IDA's association with carotid thrombosis and embolic strokes have also been reported [7].

Secondary (reactive) thrombocytosis makes up around $88 \%$ of all causes of thrombocytosis [1]. IDA is a cause of secondary thrombocytosis [1]. The exact mechanism of thrombocytosis in IDA is unclear, and several theories exist. Thrombocytosis, hypercoagulability, and hypoxia secondary to anemia are some of the mechanisms which are thought to trigger thrombosis and cause strokes in patients with IDA. It is thought that thrombopoietin is a glycoprotein hormone synthesized in the liver and kidneys which regulates the production of platelets by regulating megakaryocyte differentiation and proliferation. Megakaryocytes and platelets have $\mathrm{c}-\mathrm{Mpl}$ receptors on the surface. Unbound thrombopoietin present in plasma interacts with c-Mpl receptors, promoting the proliferation of megakaryocytes. A fall in platelet count leads to an increase in free unbound thrombopoietin which in turn interacts with $\mathrm{c}-\mathrm{Mpl}$ receptors on megakaryocytes, stimulating their maturation. Conversely, an increase in platelet count decreases free unbound thrombopoietin with a decrease in its interaction between c-Mpl receptors on megakaryocytes, decreasing maturation. IL-6 is thought to upregulate thrombopoietin messenger RNA in the liver in acute phase response. Thus interactions between IL-6, thrombopoietin, and c-Mpl receptors on megakaryocytes and platelets regulates platelet proliferation and maturation [1].

IDA is also thought to cause hypercoagulability by altering the blood flow dynamics in major vessels. If we take into account Reynolds number $=\mathrm{R}$ vD/N (where $\mathrm{R}$ is the radius of the vessel, $\mathrm{v}$ is the flow velocity, $\mathrm{d}$ is the density of blood, and $\mathrm{n}$ is the viscosity of blood), we can conclude that increased turbulent flow is caused in IDA. Reynolds number is elevated due to an increase in blood flow velocity (v) and a decrease in blood viscosity (n). The Virchow's triad of blood stasis, endothelial injury, and hypercoagulability can explain thrombosis in this scenario [7].

Anemia is associated with higher stroke mortality [8]. While anticoagulation is the mainstay of aortic arch thrombosis, surgical options can also be considered due to their safety profile in younger patients but remain contentious, as aortic arch surgery is itself a risk factor for embolic strokes [9-12].

\section{Conclusion}

An embolic phenomenon in middle-aged females with IDA warrants a TEE with an evaluation of aortic arch. If an 


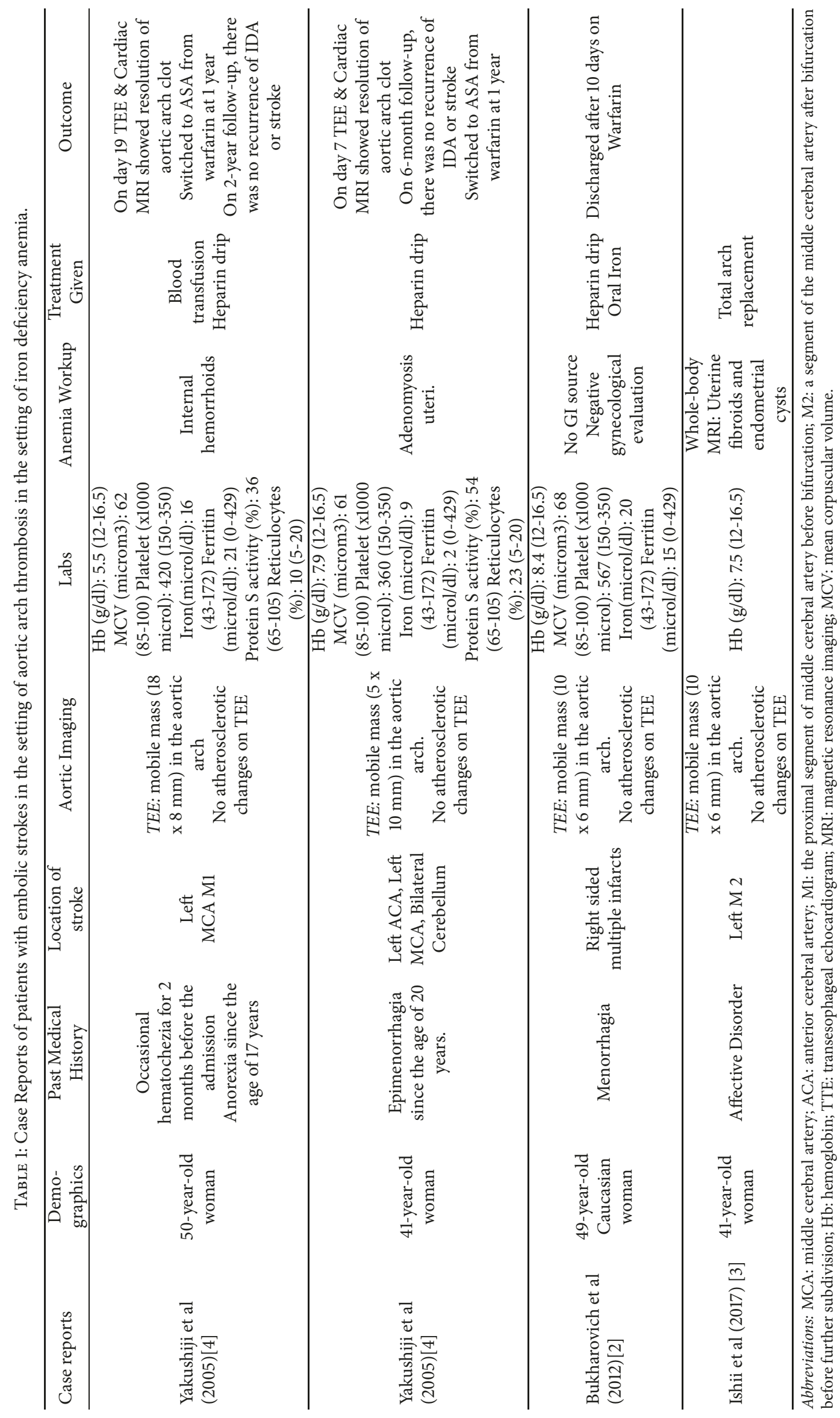


aortic arch thrombosis is discovered, the patient should be anticoagulated.

\section{Disclosure}

This case was presented as an abstract at the European Stroke Conference, 2018.

\section{Conflicts of Interest}

The authors declare that there are no conflicts of interest regarding the publication of this paper.

\section{References}

[1] A. I. Schafer, "Thrombocytosis," The New England Journal of Medicine, vol. 350, no. 12, pp. 1211-1219, 2004.

[2] I. F. Bukharovich, O. Wever-Pinzon, A. Shah, G. Todd, F. A. Chaudhry, and M. V. Sherrid, "Arterial embolism caused by large mobile aortic thrombus in the absence of atherosclerosis, associated with iron deficiency anemia," Journal of Echocardiography, vol. 29, no. 3, pp. 369-372, 2012.

[3] H. Ishii, H. Endo, and H. Kubota, "Brain infarction associate with a mobile thrombus in the aortic arch at the previous site of the ductus arteriosus," The Journal of Thoracic and Cardiovascular Surgery, vol. 154, no. 1, pp. e1-e3, 2017.

[4] Y. Yakushiji, Y. Terasaki, R. Otsubo et al., "Brain Embolism Caused by a Mobile Aortic Thrombus with Iron Deficiency Anemia," Cerebrovascular Disease, vol. 20, no. 6, pp. 475-478, 2005.

[5] P. A. Tunick and I. Kronzon, "Protruding atherosclerotic plaque in the aortic arch of patients with systemic embolization: A new finding seen by transesophageal echocardiography," American Heart Journal, vol. 120, no. 3, pp. 658-660, 1990.

[6] T. Laperche, C. Laurian, R. Roudaut, and P. G. Steg, "Mobile thromboses of the aortic arch without aortic debris: A transesophageal echocardiographic finding associated with unexplained arterial embolism," Circulation, vol. 96, no. 1, pp. 288294, 1997.

[7] P. T. Akins, S. Glenn, P. M. Nemeth, and C. P. Derdeyn, "Carotid artery thrombus associated with severe iron-deficiency anemia and thrombocytosis," Stroke, vol. 27, no. 5, pp. 1002-1005, 1996.

[8] R. S. Barlas, K. Honney, Y. K. Loke et al., "Impact of hemoglobin levels and anemia on mortality in acute stroke: analysis of UK regional registry data, systematic review, and meta-analysis," Journal of the American Heart Association, vol. 5, no. 8, 2016.

[9] H. E. Achneck, J. A. Rizzo, M. Tranquilli, and J. A. Elefteriades, "Safety of thoracic aortic surgery in the present era," he Annals of Thoracic Surgery, vol. 84, no. 4, pp. 1180-1185, 2007.

[10] R. S. Turley, J. Unger, M. W. Cox, J. Lawson, R. L. McCann, and C. K. Shortell, "Atypical Aortic Thrombus: Should Nonoperative Management Be First Line?” Annals of Vascular Surgery, vol. 28, no. 7, pp. 1610-1617, 2014.

[11] L. J. Goldstein, R. R. Davies, J. A. Rizzo et al., "Stroke in surgery of the thoracic aorta: incidence, impact, etiology, and prevention," The Journal of Thoracic and Cardiovascular Surgery, vol. 122, no. 5, pp. 935-945, 2001.

[12] T. Okada, M. Shimamoto, F. Yamazaki et al., "Insights of stroke in aortic arch surgery: identification of significant risk factors and surgical implication," General Thoracic and Cardiovascular Surgery, vol. 60, no. 5, pp. 268-274, 2012. 


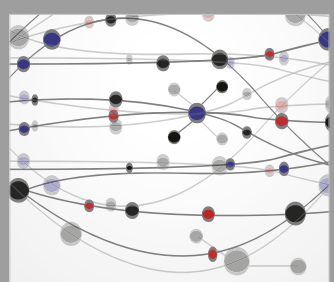

The Scientific World Journal
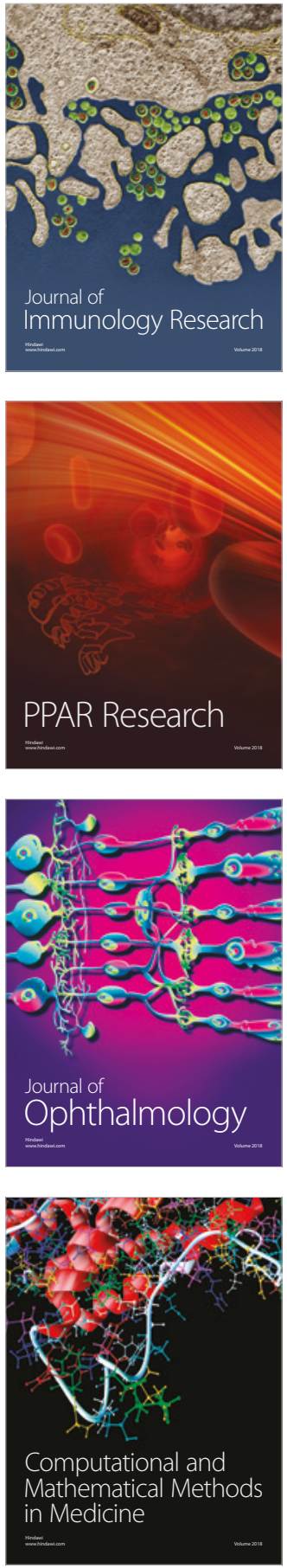

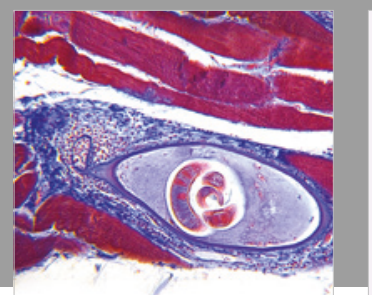

Gastroenterology Research and Practice

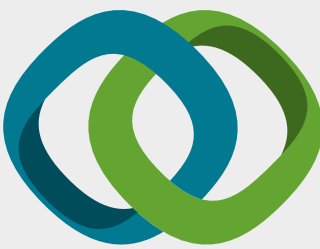

\section{Hindawi}

Submit your manuscripts at

www.hindawi.com
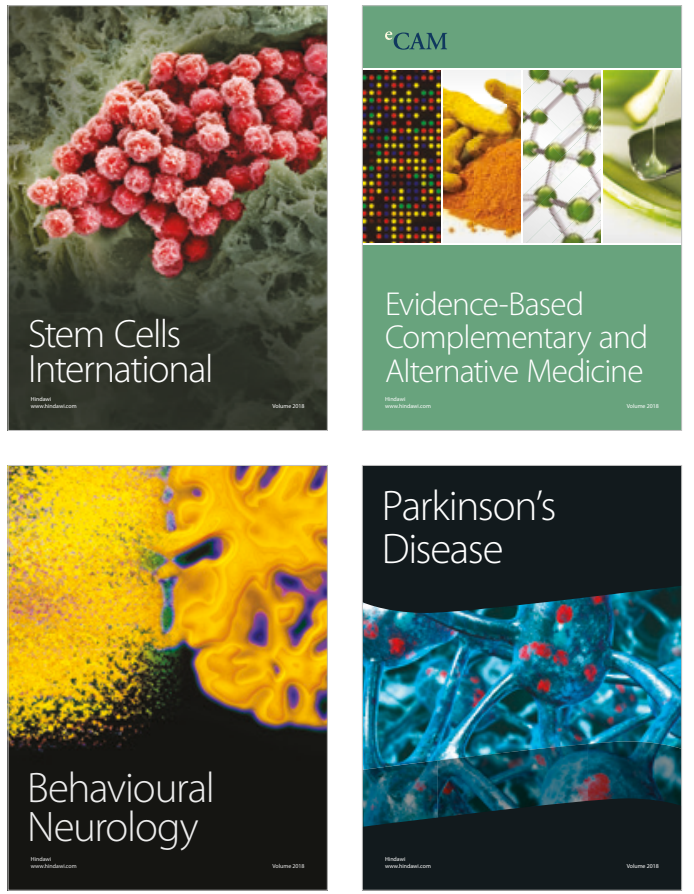

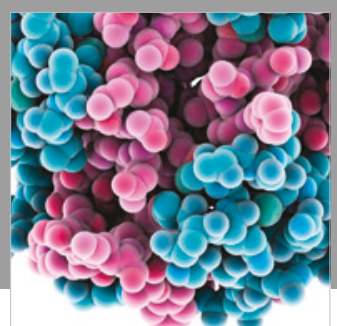

ournal of

Diabetes Research

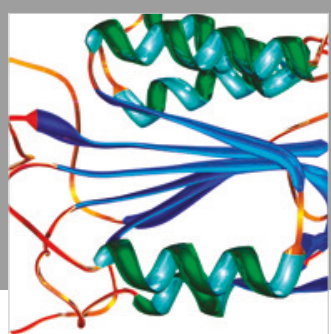

Disease Markers
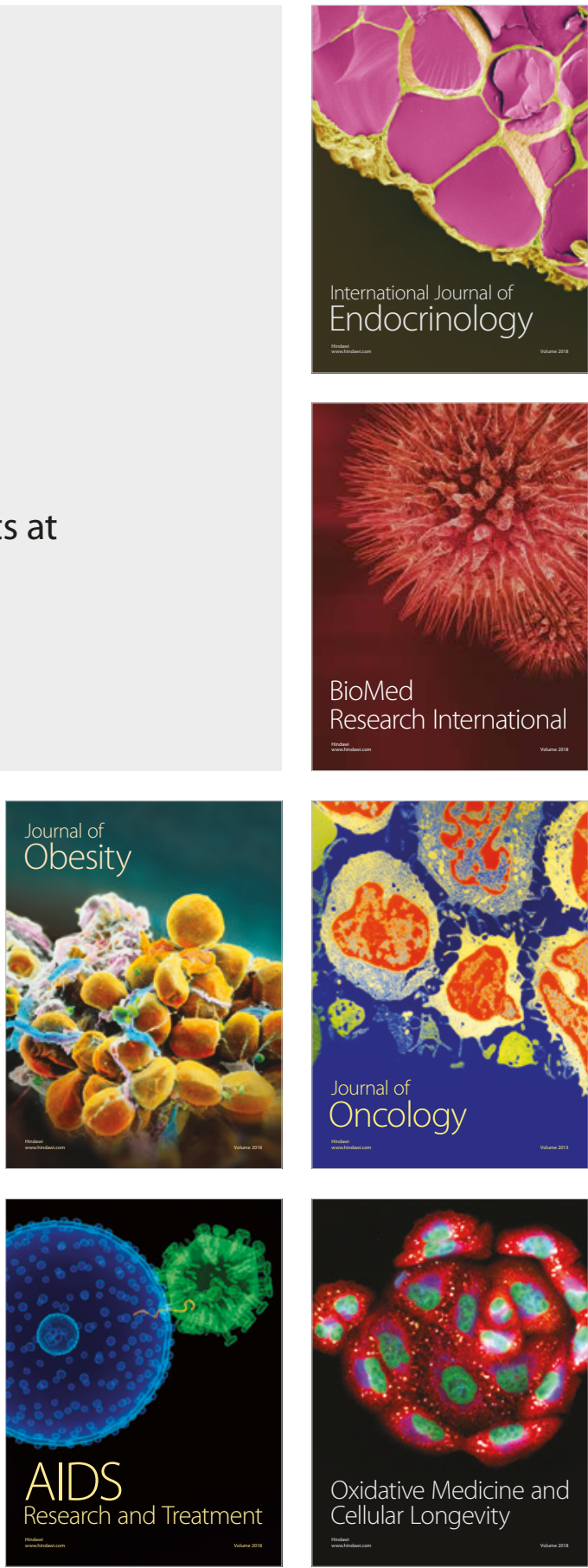